\title{
Identifying the differentially expressed microRNAs in esophagus squamous cell carcinoma of Kazakh patients in Xinjiang
}

\author{
LIYUN CHENG, GUIJUN SHI, CHUNXIAO FANG, GUANGHUA LI, YONG ZHENG and WEIGANG CHEN \\ Department of Digestion, The First Affiliated Hospital, School of Medicine, \\ Shihezi University, Shihezi, Xinjiang 832000, P.R. China
}

Received March 14, 2018; Accepted November 26, 2018

DOI: $10.3892 /$ ol.2019.9904

\begin{abstract}
Despite improvements in diagnosis and treatment, the survival of patients with advanced stages of esophageal squamous cell carcinoma (ESCC) remains poor. Therefore, novel biomarkers that can assist with early detection of ESCC are required. In the present study, three paired ESCC and normal esophageal tissue samples from Xinjiang Kazakh patients were obtained and microRNA (miRNA) microarray analysis was used to detect the differentially-expressed miRNAs. The target genes of the identified miRNAs were predicted using miRWalk software. A total of 23 miRNAs were differently expressed in Kazakh patients with ESCC. Gene Ontology enrichment analysis demonstrated that the upregulated miRNAs were predominantly associated with the 'vesicle' and 'membrane-bounded vesicle' terms, while the downregulated miRNAs were primarily associated with the term 'negative regulation of integrin-mediated signaling pathway'. The most highly enriched Kyoto Encyclopedia of Genes and Genomes pathway for the differentially-expressed miRNAs was 'Endocrine and other factor-regulated calcium reabsorption'. Protein-protein interaction network analysis revealed that IQ motif containing GTPase activating protein 1, RAB11A, lysine acetyltransferase 2B, catenin $\alpha 1$ and tight junction protein 2 were hub genes of the network. In conclusion, a number of differentially-expressed miRNAs were identified in ESCC tissues samples from Xinjiang Kazakh patients,
\end{abstract}

Correspondence to: Professor Weigang Chen or Professor Yong Zheng, Department of Digestion, The First Affiliated Hospital, School of Medicine, Shihezi University, 107 Second North Road, Shihezi, Xinjiang 832000, P.R. China

E-mail: 13579456959@126.com

E-mail: zy2850@126.com

Abbreviations: SCC, squamous cell carcinoma; ESCC, esophageal squamous cell carcinoma; miRNA, microRNA; GO, Gene Ontology; KEGG, Kyoto Encyclopedia of Genes and Genomes; PPI, protein-protein interaction; STRING, Search Tool for the Retrieval of Interacting Genes/Proteins

Key words: esophageal cancer, esophagus squamous cell carcinoma, microRNA, microarray, Kazakh which may improve the understanding of the processes of tumorigenesis and development.

\section{Introduction}

Esophageal cancer is a common type of malignant tumor of the digestive tract that originates from the esophageal epithelium or glands (1). Based on the International Agency for Research on Cancer statistics, 455,800 new esophageal cancer cases were estimated and 400,200 mortalities occurred in 2012 worldwide, which makes the incidence and mortality rates of esophageal cancer the eighth and sixth highest in the world, respectively (2). Esophageal cancer can predominantly be divided into esophageal squamous cell carcinoma (ESCC) and adenocarcinoma, and $>90 \%$ of esophageal cancer cases are classified as ESCC in China (3). Despite improvements in diagnosis and treatment, the survival rate of patients with advanced stages of ESCC remains poor. In 2014, based on a long-term follow-up survey of 21 cancer registries, the National Cancer Center estimated that the 5-year survival rate of esophageal cancer in China was $20.9 \%$ (4). The Xinjing Kazakh ethnic group is a Chinese minority with a high incidence of esophageal carcinoma (5). Studies suggest that the mortality rate of esophageal cancer for people of Kazakh ethnicity in the Xinyuan County of Xinjiang was $0.0411 \%$ between 2000 to 2005, which was much higher compared with that for individuals of the local Han ethnicity (0.0088\%) (6), which suggests ESCC poses a serious threat to the health condition of Kazakh individuals. Therefore, novel biomarkers that can assist with the early detection of ESCC in Kazakh patients are urgently required.

microRNAs (miRNAs) are a class of non-coding RNAs comprising of 19-24 nucleotides that regulate gene expression through post-transcriptional, RNA interference and gene silencing pathways (7). miRNAs can inhibit gene expression through complementary binding to the 3'-untranslated region of the target mRNAs (8). miRNAs have been demonstrated to be crucial for cancer initiation, progression and dissemination (9). It has been identified that $>50 \%$ of miRNAs are encoded in chromosome fragile sites, which are frequently absent, amplified or rearranged in malignant tumor cells, thereby resulting in the dysregulated expression of miRNAs (10). An estimated $30 \%$ of human protein-coding genes are regulated by miRNAs (11). Furthermore, miRNAs serve key roles 
in tumor progression and resistance to chemotherapy by regulating the expression of oncogenes and tumor suppressor genes (12). Previous studies have indicated that various miRNAs are associated with ESCC in Kazakh patients $(13,14)$. However, to the best of our knowledge, no previous studies have performed an analysis on the whole genome level. In recent years, the combination of high-throughput sequencing and bioinformatics analysis has made it possible to analyze the role of miRNAs that are differently expressed in ESCC in Kazakh patients. In the present study, to identify miRNA signatures in ESCC in Kazakh patients and uncover their potential mechanisms, microarray analysis was used to identify differently-expressed miRNAs between three paired ESCC and normal esophageal tissue samples from Kazakh patients. Furthermore, bioinformatic methods were used to analyze the functions of the differentially-expressed miRNAs.

\section{Patients and methods}

Clinical characteristics and tissue samples. A total of 13 samples of ESCC tissue and corresponding adjacent normal tissue were obtained from patients with esophageal squamous cell carcinoma treated surgically at the First Affiliated Hospital of Medical College of Shihezi University (Shihezi, China) from November 2014 to September 2015. Among them, three samples were used for microarray testing, including two samples obtained from males and one from a female (age range, 63-70 years). In addition, ten samples were used for reverse transcription-quantitative polymerase chain reaction (RT-qPCR) analysis, including five from males and five from females (age range, 62-75 years). No patients had a history of smoking and drinking, and no chemoradiotherapy was received prior to surgery. All tumors were stage T2N1M0 (15). All tumor samples were confirmed by two pathologists as moderately differentiated ESCC. The collection of specimens was approved by the Ethics Committee of the First Affiliated Hospital of Medical College of Shihezi University and authorized by the patients or family members. Each tissue sample was transferred to liquid nitrogen immediately following surgery and stored in a $-80^{\circ} \mathrm{C}$ freezer. All microarray experiments were completed at CapitalBio Corporation (Beijing, China).

RNA extraction and whole genome miRNA profiling. Total RNA was isolated from $\sim 2-3 \mathrm{~g}\left(2 \times 2 \times 2 \mathrm{~cm}^{3}\right)$ of tumor tissue samples and the corresponding normal tissue samples. Total RNA from frozen tumor tissue samples was extracted using an Ambion mirVana miRNA Isolation kit (Ambion; Thermo Fisher Scientific, Inc., Waltham, MA, USA), according to the manufacturer's protocol. The quality and quantity of total RNA samples were tested using a spectrophotometer. A preparation of RNA with an A260/A280 ratio of 1.8-2.0 was used for the microarray analysis. Formaldehyde denaturing gel electrophoresis was also used to test the quality of total RNA. When the $28 \mathrm{~S}: 18 \mathrm{~S}$ ribosomal $\mathrm{RNA} \geq 1: 1$, it met the experimental requirements for gene chip array analysis. Affymetrix GeneChip miRNA 4.0 Arrays (Affymetrix; Thermo Fisher Scientific, Inc.) were used to detect the miRNAs in the samples. All miRNAs were labeled with biotin using a FlashTap RNA Labeling kit (Genisphere LLC, Hatfield, PA, USA), and subsequently chip hybridization and data analysis were performed.
According to Significance Analysis of Microarrays software $\mathrm{R} 2.0$ (16), $\mathrm{q}<0.05$ and fold change $\geq 2$ or $\leq 0.5$ indicated a statistically significant differentially expressed miRNA.

Prediction of miRNA targets and functional enrichment analysis. The target genes of the identified miRNAs were predicted using miRWalk (http://mirwalk.uni-hd.de/), which includes 12 databases, including TargetScan, miRanda and MiRmap. Gene Ontology (GO) analysis and Kyoto Encyclopedia of Genes and Genomes (KEGG) pathway analysis were applied using KOBAS 2.0 (http://kobas.cbi. pku.edu.cn.). Fisher's exact test was performed to classify the GO terms and KEGG pathways, and $\mathrm{P}<0.05$ indicated a statistically significant GO term or KEGG pathway.

Generation of a protein-protein interaction (PPI) network. Search Tool for the Retrieval of Interacting Genes/Proteins (STRING) database (https://string-db.org/cgi/input.pl) is an online tool for analyzing protein-protein interaction information. To analyze the interactions of the target genes, the genes were mapped to the STRING database and a PPI network was constructed. Cytoscape 3.6.0 (17) was used to visualize the PPI network and identify the hub genes. The node degrees of the target genes were analyzed and the first five were selected as the network's hub genes.

Integration of miRNA-mRNA co-expression network. The correlation test within the 'stats' package in $\mathrm{R} 2.15 .3$ (http://www.R-project.org/) was used for the co-expression analysis. According to the principle of negative interaction between miRNA and mRNA, a miRNA-mRNA co-expression network was integrated using $\mathrm{P}<0.05$ and correlation coefficient $<-0.99$, Pearson's correlation coefficient was used for the correlation analysis. Cytoscape software was then used to generate a co-expression network.

$R T$ - $q P C R$. An additional 10 Kazakh ESCC tissue samples and corresponding adjacent normal tissue samples were obtained for RT-qPCR verification. Total RNA extraction was performed using the aforementioned method. Subsequently, a miRcute miRNA First-Strand cDNA Synthesis kit and a miRcute miRNA qPCR Detection kit (SYBR ${ }^{\circledR}$ Green; both from Tiangen Biotech Co., Ltd., Beijing, China) were used to detect the levels of miRNA, according to the manufacturer's protocols. U6 was used as an internal control. The RT reaction system $(20 \mu \mathrm{l})$ contained $2 \mu \mathrm{l}$ total RNA, $10 \mu \mathrm{l} 2 \mathrm{X}$ miRNA RT reaction buffer, $2 \mu 1$ miRNA RT enzyme mix and $6 \mu \mathrm{l}$ RNase-free ddH2O. The reactions were performed at $42^{\circ} \mathrm{C}$ for $60 \mathrm{~min}$ and $95^{\circ} \mathrm{C}$ for $3 \mathrm{~min}$. The $20 \mu \mathrm{l}$ reaction system for qPCR included $10 \mu \mathrm{l}$ 2X miRcute Plus miRNA Premix (with SYBR and ROX), $1 \mu 1$ forward primer, $0.4 \mu \mathrm{l}$ reverse primer $(10 \mu \mathrm{M}), 2 \mu \mathrm{l}$ first-strand

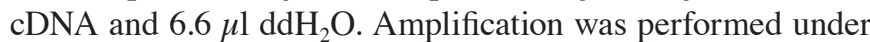
the following reaction conditions: $95^{\circ} \mathrm{C}$ for $15 \mathrm{~min}$, followed by 40 cycles of $94^{\circ} \mathrm{C}$ for $20 \mathrm{sec}$ and $60^{\circ} \mathrm{C}$ for $34 \mathrm{sec}$. qPCR was performed on a CFX96 Real-Time system (Bio-Rad Laboratories, Inc., Hercules, CA, USA) using the following forward primers: U6, 5'-GCTTCGGCAGCACATATACTA AAAT-3'; miR-664b-5p, 5'-TGGGCTAAGGGAGATGATTGG GTA-3'; miR-767-5p, 5'-TGCACCATGGTTGTCTGAGCA TG-3'; miR-223-3p, 5'-GCGTGTCAGTTTGTCAAATACCCC 


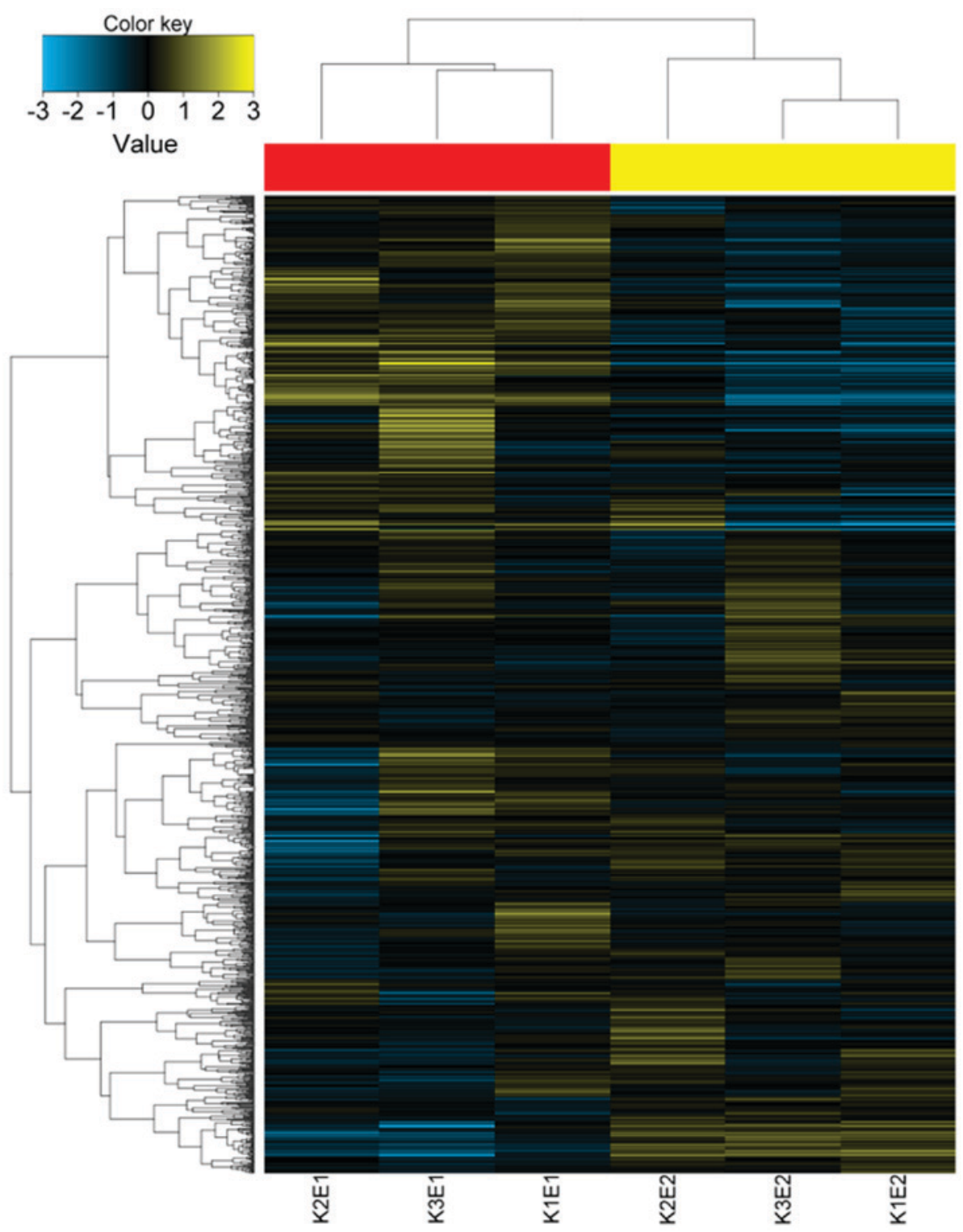

Figure 1. Heat map of differentially expressed microRNAs in Kazakh esophagus squamous cell carcinoma tissues. Yellow represents upregulation and blue represents downregulation. Samples K1E1, K2E1 and K3E1 represent three cases of ESCC tissue. Samples K1E2, K2E2 and K3E2 represent the corresponding normal tissues.

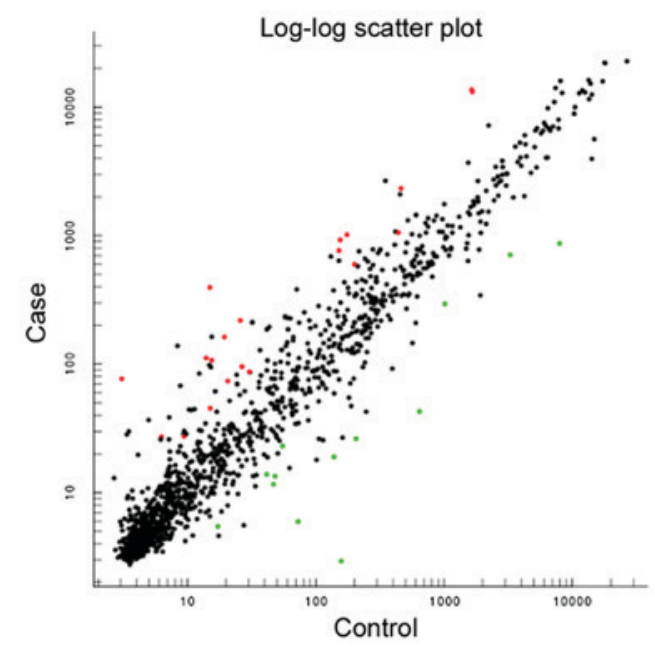

Figure 2. Scatter plot of differentially expressed microRNAs in Kazakh esophagus squamous cell carcinoma tissues. Red represents upregulation and green represents downregulation.
A-3'; miR-203a, 5'-cgcAGTGGTTCTTAACAGTTCAACAGT T-3'; miR-373, 5'-GACGAGCCCCTCGCACAAAC-3'. The reverse primers were contained in the miRcute miRNA qPCR Detection kit. Relative quantification of miRNA expression was calculated using the $2^{-\Delta \Delta \mathrm{Ct}}$ method (18) the data were statistically analyzed by Student's t-tests, and $\mathrm{P}<0.05$ was considered to indicate a statistically significant differences between groups. For each sample, all experiments were performed in triplicate.

\section{Results}

Identification of differentially-expressed miRNAs. To determine the miRNA expression patterns in ESCC tissue samples and their corresponding normal tissue samples, miRNA microarray analysis was conducted. A total of 23 miRNAs were identified to be significantly differentially expressed between the two groups; 12 miRNAs were upregulated and 11 miRNAs were downregulated. As demonstrated in 
Figs. 1 and 2, the expression of these miRNAs differed between Kazakh ESCC and matched normal tissues. The differentially expressed-miRNAs are displayed in Table I.

Target gene prediction. miRWalk 2.0 was used to predict the target genes of the differentially-expressed miRNAs. Of the 23 differentially-expressed miRNAs, 224 target genes were predicted for 21 miRNAs, as presented in Table II.

KEGG pathwayanalysis.Using KEGG pathway analysis, a total of five terms were enriched among the upregulated miRNAs and eight terms were enriched among the downregulated miRNAs. Table III presents the significantly enriched pathways of the differently-expressed miRNAs. The upregulated miRNAs were identified to be predominantly involved in the following pathways: 'Glycosphingolipid biosynthesis-ganglio series' and 'Endocrine and other factor-regulated calcium reabsorption'. The downregulated miRNAs were revealed to be involved in the following pathways: 'Endocrine and other factor-regulated calcium reabsorption', 'Lysine degradation' and 'Steroid biosynthesis'.

GO term enrichment analysis. To further understand the associated functions of the differently-expressed miRNAs, $\mathrm{GO}$ analysis was performed. GO enrichment analysis included the categories cellular component, biological process and molecular function. A total of 750 terms were enriched for the upregulated miRNAs (Fig. 3) and 888 terms were enriched for the downregulated miRNAs (Fig. 4).

Protein-protein interaction (PPI) network analysis. Based on the information in the STRING database, a PPI network (Fig. 5) of the target genes was generated. Cytoscape software analysis of the target genes in the network revealed a maximum node degree of seven and a minimum of one. The following top five hub genes were selected: IQ motif containing GTPase activating protein 1 (IQGAP1), RAB11A, lysine acetyltransferase 2B (KAT2B), catenin $\alpha 1$ (CTNNA1) and tight junction protein 2 (TJP2).

Integration of miRNA-mRNA co-expression network. According to the principle of negative interaction between miRNA and mRNA, miRNAs and mRNAs with a correlation coefficient $<-0.99$ were selected to establish the miRNA-mRNA co-expression network using Cytoscape software. The network comprised of 273 nodes and 326 edges. As depicted in Fig. 6 , the network consisted of 21 miRNAs (11 upregulated and 10 downregulated) and $252 \mathrm{mRNAs}$. The co-expression network demonstrates that the mRNAs may be potential target genes of the differently-expressed miRNAs and the mRNAs may be involved in the occurrence and development of ESCC.

Identification of final target genes of differentially expressed miRNAs. If the target mRNAs predicted by miRWalk also appeared in the co-expression network they were designated as the final target genes. A total of $25 \mathrm{mRNAs}$ and 11 miRNAs were presented in the co-expression network (Fig. 7).

Validation of microarray data using RT-qPCR. To validate the microarray data, RT-qPCR was performed to detect the
Table I. Expression of microRNAs in Kazakh esophagus squamous cell carcinoma tissues and corresponding normal tissues.

\begin{tabular}{lccl}
\hline Gene name & Fold change & q-value & Regulation \\
\hline hsa-miR-664b-5p & 26.4885 & 0.0316 & $\mathrm{Up}$ \\
hsa-miR-767-5p & 25.0685 & 0.0316 & $\mathrm{Up}$ \\
hsa-miR-1301-3p & 8.5011 & 0 & $\mathrm{Up}$ \\
hsa-miR-93-3p & 8.3847 & 0 & $\mathrm{Up}$ \\
hsa-miR-21-3p & 7.9849 & 0 & $\mathrm{Up}$ \\
hsa-miR-25-5p & 6.9362 & 0.0195 & $\mathrm{Up}$ \\
hsa-miR-3651 & 5.9744 & 0 & $\mathrm{Up}$ \\
hsa-miR-99b-5p & 5.0415 & 0.0316 & $\mathrm{Up}$ \\
hsa-miR-223-3p & 4.3435 & 0 & $\mathrm{Up}$ \\
hsa-miR-505-5p & 3.5862 & 0 & $\mathrm{Up}$ \\
hsa-miR-1273h-5p & 3.0283 & 0 & $\mathrm{Up}$ \\
hsa-miR-423-5p & 2.4030 & 0.0195 & $\mathrm{Up}$ \\
hsa-miR-6086 & 0.4195 & 0.0397 & Down \\
hsa-miR-3663-3p & 0.3356 & 0.0373 & Down \\
hsa-miR-378e & 0.3160 & 0.0373 & Down \\
hsa-miR-5100 & 0.2901 & 0.0498 & Down \\
hsa-miR-1273g-3p & 0.2163 & 0 & Down \\
hsa-miR-422a & 0.1372 & 0.0397 & Down \\
hsa-miR-1224-5p & 0.1277 & 0.0373 & Down \\
hsa-miR-203a & 0.1094 & 0.0397 & Down \\
hsa-miR-617 & 0.0818 & 0 & Down \\
hsa-miR-375 & 0.0666 & 0.0373 & Down \\
hsa-miR-5580-3p & 0.0186 & 0 & Down \\
\hline
\end{tabular}

expression level of certain selected differentially-expressed miRNAs, including miR-664b-5p, miR-767-5p, miR-223-3p, miR-203a and miR-375. The RT-qPCR results and microarray data were consistent (Fig. 8).

\section{Discussion}

The incidence of esophageal cancer is not only associated with environmental factors, living habits, microorganisms and other external factors, but also closely associated with genetic factors and differential gene expression (19). Therefore, it is important to investigate the differentially-expressed genes involved in the pathogenesis of esophageal carcinoma. Microarray and high-throughput sequencing technology are beneficial methods for detecting the expression of thousands of genes simultaneously; therefore, these methods are frequently used to investigate potential biological targets for the diagnosis and treatment of esophageal cancer $(20,21)$. Although numerous studies have been devoted to the differential expression of genes associated with esophageal cancer in Kazakh patients $(22,23)$, to the best of our knowledge, the expression of miRNAs has not been systematically evaluated. In the present study, the differentially-expressed miRNAs in Xinjiang Kazakh cases of ESCC were identified by microarray analysis and their biological functions were preliminarily analyzed. This may enrich the investigation of the gene expression profiles in Kazakh ESCC. 
Table II. Target genes of differentially expressed miRNAs.

miRNA
hsa-miR-99b-5p
hsa-miR-93-3p
hsa-miR-767-5p
hsa-miR-664b-5p

hsa-miR-505-5p

hsa-miR-423-5p

hsa-miR-3651

hsa-miR-25-5p

hsa-miR-223-3p

hsa-miR-21-3p

hsa-miR-1301-3p

hsa-miR-1273g-3p

hsa-miR-1224-5p

hsa-miR-617
Target gene

Expression

N4BP3

ABLIM1, AMFR, C6orf132, CCNG2, COL4A3BP, CYP11A1, FAM46B, FYCO1, GANC, GIPC1, H2AFY, IKZF2, KLF8, KLHL2, LCOR,LYNX1, MUC1, N4BP3, NFIA, PRNP, RMND5B, SIM2, SLC12A7, SORT1, TMEM201, TPCN1, TRPS1 ABLIM1, ARSB, ATP1B1, CCNG2, CD276, CDH3, CHIC2, CUL3, CYP24A1, ELOVL6, ERAP1, FAM126B, FNDC3B, FUT2, GABRP, GANC, GLI2, IQGAP1, LAMC2, LCOR, NDST1, NFIA, PHACTR4, PI15, PIK3C2A, PPL, PRDM1, RAB11A, SCNN1B, SDK1, SEC24A, SERAC1, SFXN3, SH3TC2, TET2, TGIF2, TJP2, TMEM79, TRPS1, ZBTB43

NFIA, LCOR, TGIF2

AGFG2, AIF1L, CAPN5, CCDC120, CD276, CDK18, EPN3, FAM189A2, FMO2, GABRP, GANC, GDI1, IL1RN, KCNQ2, KIAA0232, KIF1C, KREMEN1, TET2, MARK2, N4BP3, NDST1, NT5C2, OCLN, PITPNM3, PRNP, RARG,RMND5B, SCP2, SDK1, SLC25A23, SLC2A3, SMAGP, SORT1,TGIF2, TMPRSS2, UNC13B ARSB, ABR, ADCY6, AGFG2, ANXA9, ARHGAP32, ARHGEF10L, B4GALNT1, BAG1, CACNG4, CAPN5, CD276, CSF1, CUL3, CYP4F22,DIAPH1, ELF3, ELOVL6, EMP1, ESRP2, FAM129B, FBXO34, FOSL2, FYCO1,GANC, GATSL3, GDI1, GPX3, HEXIM1, HLF, KREMEN1, LCOR, LYNX1,MAP3K9, MARK2, MGLL, MKNK2, N4BP3, NAT14, NCCRP1, NDST1, NPR3,PAQR8, PDLIM5, PI15, PIM1, PMM1, PRDM1, PTK6, RAB25, RABGAP1L,RALB, RFFL, SAR1B, SDK1, SH3TC2, SLC25A23, SLC2A3, SLC35C1, SORT1,ST6GALNAC6, THRB, TMEM144, TMEM201, TOM1, TPCN1, TRPS1, TYRO3, VAT1, VAV3

COBLL1, FBXO34, IKZF2, MGLL, NFIA, SLC17A5, ST3GAL4, TRIM13, VAV3 ABR, BNIPL, EXPH5, FAM114A1, FUT2, MARK2, KIF1C, OCLN, PHACTR4, TGIF2, TRIM9, ZNF185

ABLIM1, ACPP, AIM1, ATP1B1, C1orf116, CAMSAP1, CAPN14, CDKN2AIP, CDOCK9, FAM219B, HEXIM1, HLF, LAMC2, LCOR, LIN54, LNPEP, TAOK3, RALGPS2, MSMO1, NCCRP1, NFIA, PIK3C2A, PLD1, PRDM1, RAB11FIP1, ABGAP1L, RASEF, SFXN3, SH3TC2, SIM2, SLC26A2, TRPS1, TWF1, DS1 AMFR, ATP1B1, CAMSAP1, CAPN5, CD46, CDK18, COBLL1, CUL3, WDR47, FAM135A, FAM219B, FNDC3B, FYCO1, HLF, LCOR, LIN54, LYNX1, STAU2, MGLL, MLLT4, NPR3, OCLN, PCYOX1, PI15, PLOD1, PMM1, PRDM1, TET2, PRKCH, RBM47, ROBO2, SAR1B, SLC26A2, ST6GALNAC6

AGFG2, ARHGAP32, BACH1, CAMSAP1, CAPN14, CCNG2, CD276, MOXD1, CLCN3, CXCL1, DIAPH1, ELF3, EPN3, FAM114A1, FAM219B, CD46, FYCO1, GANC, GRHL1, IKZF2, KLHL2, KREMEN1, LAMB3, LCOR, PRDM1, SORT1, FUT2, LIN54, LYNX1, MMP11, FBXO34, TET2, NAPA, NDST1, NFIA, TRPS1, NT5C2, PAQR8, PCSK5, PITX1, PRNP, RBM47, ROBO2, SAR1B, VAV3, TOM1, SASH1, SH3TC2, SIM2, SLC25A23, SLC26A2, ZBTB43, TMEM108, TOM1L2, TMEM201, TRIM9, NPR3,

ABLIM1, ARSB, CAMSAP1, CCNG2, CLCN3, GNE, GOLGA2, LIN54, NFIA, PCYOX1, PRDM1, RABGAP1L, RFFL, SDK1, SH3TC2

ABLIM1, AIF1L, ALDH9A1, B4GALNT1, CAPN5, CD207, ZBTB43, SLC2A3, CYP4F22, FAM129B, FBXO34, FMO2, FNDC3B, FYCO1, HEXIM1, USP54, KAT2B, KIAA0232, PITPNM3, LCOR, MARVELD3, MOXD1, NAT14, NDRG2,PAQR8, PCYOX1, PHACTR4, TMEM108, PITX1, RAI1, RBM47, SCP2, SDK1,SLC35C1, SNX33, TMPRSS11B, TRIM13, TRPS1, UPRT, EPN3, THRB, CUL3, DLX1, BAG1 ABHD17B, AMFR, ARHGAP32, CAPN5, CCDC150, CDS1, CUL3, ERAP1, FAM219B, FNDC3B, GABRP, GRHL1, HES5, HLF, HOOK3, KANK1, LYNX1, NDST1, NFIA, OCLN, PCYOX1, PRKCH, RAB11FIP1, RALGPS2, SEC24A, SH3TC2, SLC26A2, SORT1, TET2, TGIF2, TMEM201

Down 
Table II. Continued.

\begin{tabular}{|c|c|c|}
\hline miRNA & Target gene & Expressio \\
\hline hsa-miR-6086 & MARK2, GIPC1 & Down \\
\hline hsa-miR-5580-3p & $\begin{array}{l}\text { ARHGAP32, ATP1B1, ERAP1, ESRP1, FAM126B, FMO2, FNDC3B, SPTLC1, } \\
\text { KLF8, MLLT4, GNE, PCYOX1, PI15, PIK3C2A, PRDM1, PRKCH, TMEM108, } \\
\text { SAR1B, SH3TC2, SLC2A3, NFIA, OCLN, RASEF }\end{array}$ & Down \\
\hline hsa-miR-5100 & $\begin{array}{l}\text { ANXA9, ARHGAP32, BARX2, EXPH5, FAM114A1, KANK1, KLB, NPR3, } \\
\text { RAB11A, RALB, PRKCH, PHACTR4 }\end{array}$ & Down \\
\hline hsa-miR-422a & $\begin{array}{l}\text { ABR, ALDH3A2, AMFR, CCNG2, COL4A3BP, CTNNA1, CUL3, DLX1, ESRP2, } \\
\text { VAT1, C1orf116, GOLPH3L, HOOK3, IKZF2, KLF8, LIN54, MOXD1, SH3TC2, } \\
\text { NDST1, NR1H2, PAQR8, PCYOX1, PEBP1, PI15, PIM1, PIM2, PLOD1, PRKCH, } \\
\text { RAB11A, SLC2A3, SLC35C1, TWF1, TRAF6, FYCO1 }\end{array}$ & Down \\
\hline hsa-miR-378e & CCNG2, GOLPH3L, PEBP1, PRKCH, RAB11A, SH3TC2, SLC2A3, TWF1 & Down \\
\hline hsa-miR-375 & $\begin{array}{l}\text { ABLIM1, ADCY6, AIM1, ATP1B1, BAG1, CACNG4, CAST, CLCN3, SERAC1, } \\
\text { COBLL1, CXCL1, CXCR2, ELOVL6, FAM135A, FMO2, FNDC3B, RMND5B, } \\
\text { IKZF2, KLF8, N4BP3, NFIA, NPR3, NT5C2, OCLN, PCYOX1, PRDM1, } \\
\text { SAMD9, RAB11A, RALGPS2, RBM47, PLD1, SLC25A23, CDS1, GANC, } \\
\text { ROBO2, THRB, TMEM144, TRPS1, UNC13B, TGIF2, SEC24A }\end{array}$ & Down \\
\hline hsa-miR-203a & $\begin{array}{l}\text { ABLIM1, ACPP, AIF1L, AIM1, ALDH3A2, ANKRD44, ARHGAP32, ARSB, } \\
\text { BAG1, CCNG2, CAPN14, CD46, CITED2, CLCN3, COBLL1, CUL3, CYP24A1, } \\
\text { DUSP5, ELOVL6, HEXIM1, EMP1, GNE, EPN3, ERAP1, FAM114A1, FYCO1, } \\
\text { FAM126B, FMO2, FNDC3B, FOSL2, GOLPH3L, GRHL3, HLF, HOOK3, IKZF2, } \\
\text { LCOR, LIN54, MLLT4, MSMO1, GLTP, N4BP3, NPR3, OVOL1, PAQR8, } \\
\text { PHACTR4, PI15, PIK3C2A, PITPNM3, PLD1, RAB11A, SEC24A, SDK1, VAV3, } \\
\text { RABGAP1L, RALB, RBM47, ROBO2, SAR1B, SASH1, SCEL, SCP2, ZNF571, } \\
\text { ZNF185, SH3TC2, SLC25A23, SLC26A2, SLC2A3, TET2, THRB, TMEM154, } \\
\text { TRPS1, TWF1, VAT1, NFIA, ZNF442, WDR47 }\end{array}$ & Down \\
\hline
\end{tabular}

miRNA, microRNA.

Table III. Top Kyoto Encyclopedia of Genes and Genomes enrichment terms for the differentially expressed microRNAs.

\begin{tabular}{lccc}
\hline Term & ID & P-value & Regulation \\
\hline Glycosphingolipid biosynthesis-ganglio series & hsa00604 & 0.012 & Up \\
Endocrine and other factor-regulated calcium reabsorption & hsa04961 & 0.014 & 0.020 \\
Steroid biosynthesis & hsa00100 & 0.032 & Up \\
Glycosphingolipid biosynthesis-lacto and neolacto series & hsa00601 & 0.040 & 0.015 \\
Thyroid hormone synthesis & hsa04918 & 0.018 & Up \\
Endocrine and other factor-regulated calcium reabsorption & hsa04961 & 0.020 & Down \\
Lysine degradation & hsa00310 & 0.025 & Down \\
Steroid biosynthesis & hsa004144 & Down \\
Endocytosis & hsa00340 & Down \\
Histidine metabolism & & &
\end{tabular}

The present study identified 23 differentially-expressed miRNAs between ESCC and normal control tissues. Among these miRNAs, certain miRNAs have previously been identified to be differentially expressed in esophageal cancer. Li et al (24) revealed that miR-375 was frequently downregulated in ESCC and was significantly associated with advanced clinical stage, tumor metastasis and poor prognosis, which indicates that miR-375 may serve as a potential biomarker to predict the outcome of ESCC. In ESCC tissue obtained from Kazakh patients, the expression of miR-375 was also identified to be downregulated in another study (25), which is consistent with the present findings. Therefore, it could be indicated that miR-375 acts as a tumor suppressor in ESCC. Wang et al (26) reported that miR-3651 was downregulated in ESCC of 


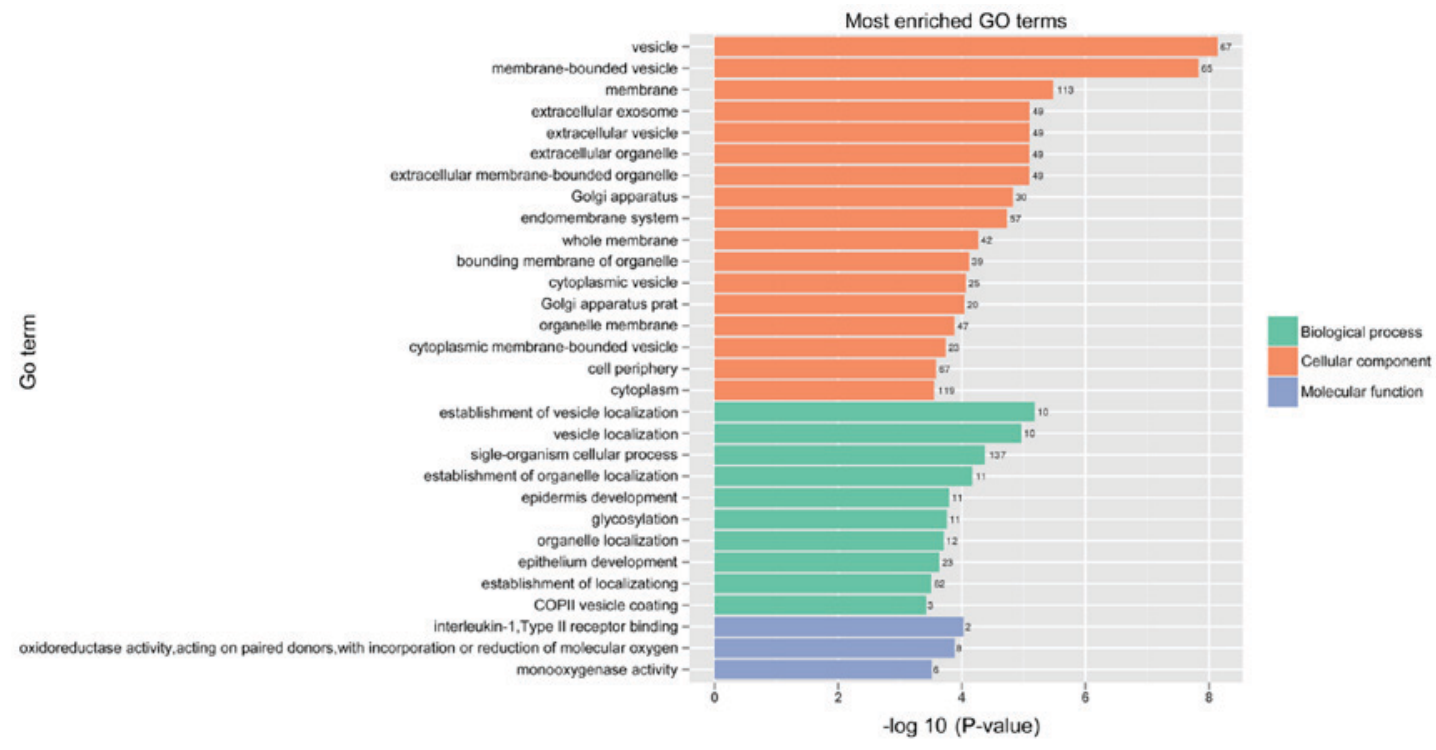

Figure 3. Top $20 \mathrm{GO}$ enrichment terms for the upregulated microRNAs. GO, Gene Ontology.

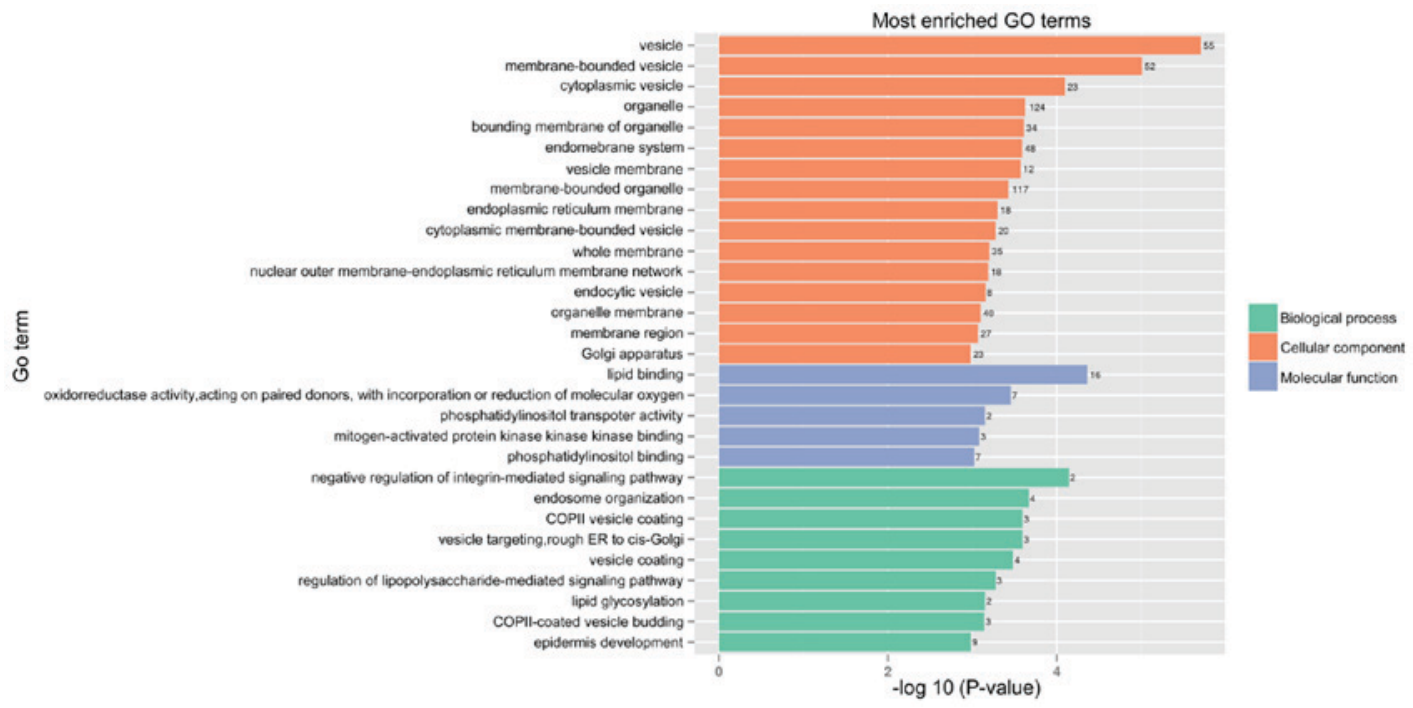

Figure 4. Top $20 \mathrm{GO}$ enrichment terms for the downregulated microRNAs. GO, Gene Ontology.

Han patients, and was negatively correlated with $\mathrm{T}$ stage and tumor size. However, in the present study it was identified that miR-3651 was upregulated. This difference may be explained by differences between the sample populations. Han and Kazakh patients may have different miRNA expression profiles, and miR-3651 may serve a different role in ESCC in patients of the two ethnicities. miR-203 has been demonstrated to be downregulated in a number of malignant tumor types, including laryngeal squamous cell carcinoma (27), hepatocellular carcinoma (28), cervical cancer (29) and lung cancer (30). Similar to the current study, Li et al (31) demonstrated that miR-203a is downregulated in ESCC samples and the transfection of miR-203a in cells mimicking esophageal cancer inhibited their proliferation and invasive capacity, indicating that miR-203a serves a tumor suppressor role. Several studies have identified that miR-21-3p is upregulated in ESCC tissue samples, which indicates miR-21-3p may serve as an oncogene by participating in the invasion and metastasis of esophageal cancer (32). Similar to the present study, Liu et al (33). reported that miR-21 is highly expressed in esophageal cancer in patients of Kazakh ethnicity, and serves a negative role in regulating programmed cell death. miR-25, a member of the miR-106b-25 cluster (34), has been identified to be overexpressed in several cancer types, including small cell lung, gastric and liver cancer (31,35-37). Xu et al (38) demonstrated that high expression of miR-25 is associated with lymph node metastasis and Tumor-Node-Metastasis stage in patients with ESCC. This study suggests that miRNA-25 is upregulated in Kazakh ESCC and may serve a role as an oncogene.

Furthermore, certain differentially-expressed miRNAs identified in the present study have previously been reported in other various cancer types, including breast cancer, glioma, lung cancer and hepatocellular carcinoma (39-43), but have not been previously identified in Kazakh ESCC. These miRNAs may serve important roles in the development and progression of ESCC. Additionally, to the best of our knowledge, certain 


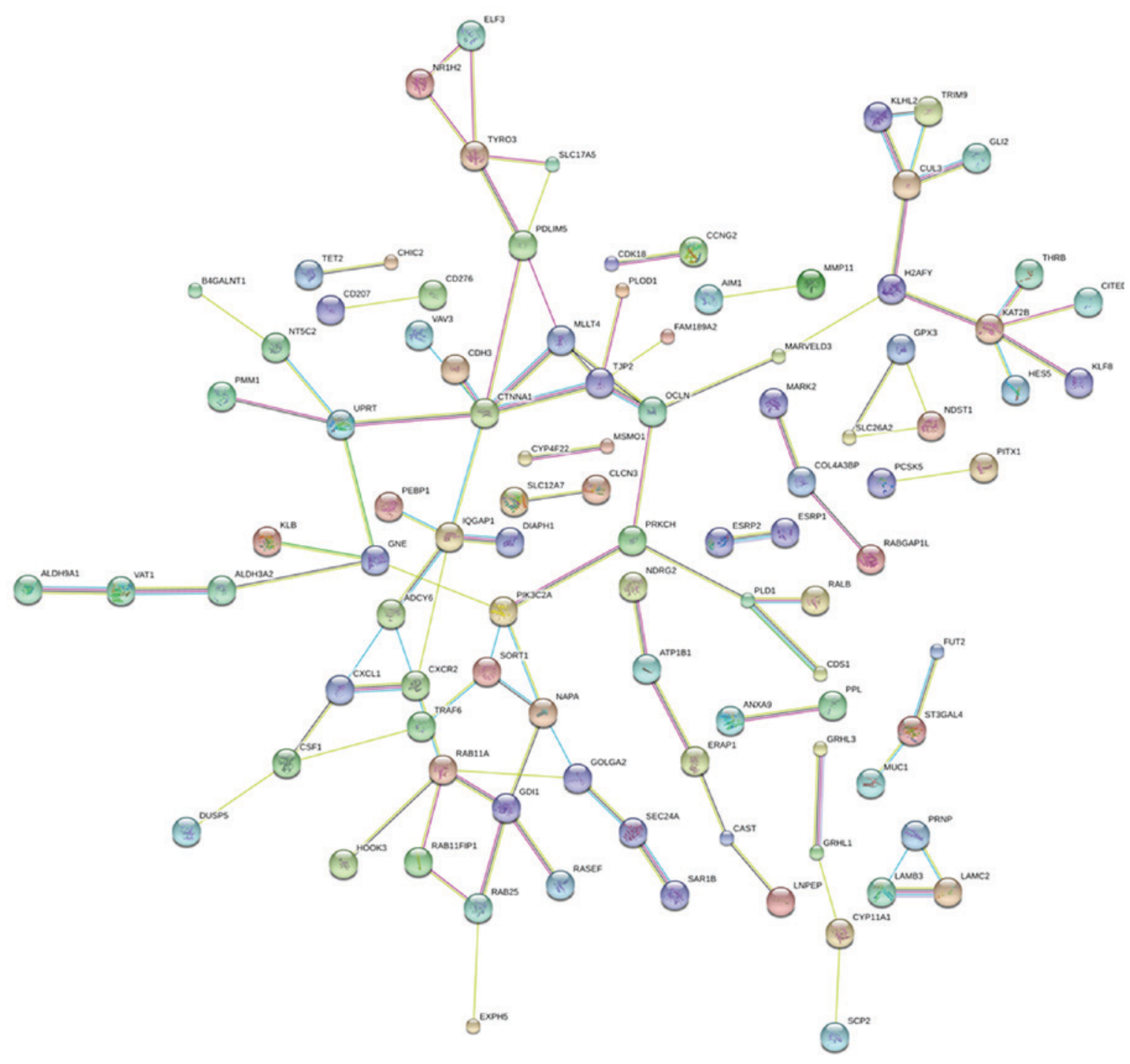

Figure 5. Protein-protein interaction network of the target genes.

miRNAs revealed in the present study have not previously been reported in any tumor types, therefore these may be potential novel markers of Kazakh ESCC. Further experiments are required to verify this observation. Additionally, RT-qPCR was used to detect the expression levels of certain miRNAs, including miR-664b-5p, miR-767-5p, miR-223-3p, miR-203a and miR-375, which were identified to be differentially expressed using microarray analysis. These results confirmed that RT-qPCR and gene chip detection were in accordance, which supports the accuracy of the microarray results.

The number and type of differentially expressed miRNAs in the current study varies from previous studies $(20,44)$. A number of factors can affect the results of microarray analysis, including the homogeneity of the sample and differences in the chips used. The current study maximized homogeneity in the selection of samples. All patients were diagnosed with primary ESCC. No patients had a history of smoking and drinking, and no chemoradiotherapy was received prior to surgery. All cancer specimens were confirmed as moderately differentiated ESCC and the tumor staging was T2N1M0. It can be hypothesized that differences with previous studies may be associated with differences in the number of samples, the region the samples were acquired from, experimental conditions, research methods, the specific chips used and the data analysis methods. Furthermore, due to different genetic backgrounds, the gene expression profile of ESCC may be different in Kazakh patients compared with Han patients. Therefore, studying the specific markers of Kazakh ESCC is important for understanding its pathogenesis.

KEGG analysis demonstrated that the differentially expressed miRNAs were mainly involved in 'glycolipid biosynthesis', 'endocrine and other glycolipid-related factors regulating calcium reabsorption' and 'steroid biosynthesis'. According to a previous study, abnormal expression of glycolipids in tissues and organs may lead to carcinogenesis (45), and the abnormal metabolism of steroids is closely associated with the occurrence, metastasis and treatment of tumors (46). Calcium ion is an important second messenger within the cell. It can participate in the proliferation, differentiation, metastasis and apoptosis of tumors in numerous ways. Therefore, the abnormal absorption of calcium ions in cells may be closely associated with tumorigenesis (47). GO analysis indicated that the upregulated miRNAs were mainly associated with vesicles and exocrine bodies. By contrast, 


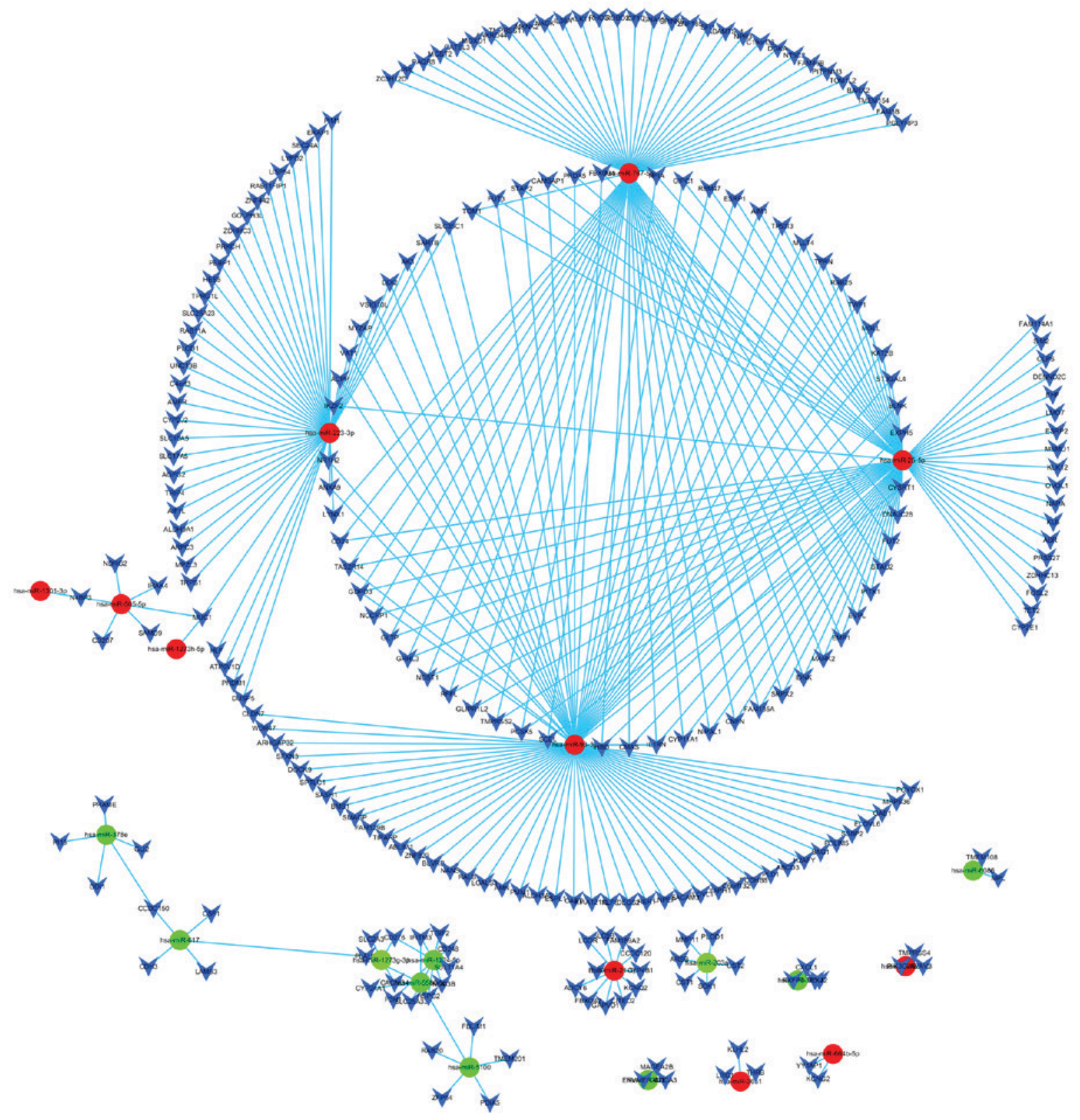

Figure 6. Co-expression network of dysregulated miRNAs and mRNAs in Kazakh esophagus squamous cell carcinoma. Red circles represent the upregulated miRNAs, green circles represent the downregulated miRNAs, blue arrows represent target genes and blue lines indicate negative correlations. miRNA, microRNA.

the downregulated miRNAs were mainly associated with the negative regulation of integrin-mediated signaling pathways, lipid binding and other functions. The exosecretion (48) and integrin-mediated signaling pathways (49) have previously been confirmed to be associated with tumors. The upregulation or downregulation of these functions may directly or indirectly lead to the occurrence and development of tumors, which suggests the differentially expressed miRNAs associated with these biological processes may be associated with ESCC. However, the specific mechanisms remain to be further elucidated.

As demonstrated in the PPI network, IQGAP1, RAB11A, KAT2B, CTNNA1 and TJP2 were identified as hub genes. IQGAP1 is a scaffolding protein that can regulate several distinct signaling pathways (50). Accumulating evidence has demonstrated that IQGAP1 serves an important role in tumorigenesis and tumor progression. Andl et al (51) demonstrated that IQGAP1 was overexpressed in ESCC tumor tissues and its overexpression was correlated with the invasion depth of ESCC. Therefore, IQGAP1 may be important in regulating ESCC occurrence and progression and may be useful in developing a novel anticancer therapy. Xu et al (52) identified that RAB11A was upregulated in ESCC and may serve a critical role in the development of ESCC. Other studies have demonstrated that KAT2B, CTNNA1 and TJP2 are important in multiple tumor types, including hepatocellular carcinoma, cervical cancer and breast cancer (53-55), however their roles in ESCC are not clear. In the current study, these three genes were targeted by the differentially expressed miRNAs in Kazakh ESCC, which suggests that they may also serve an important role in the development of ESCC. 


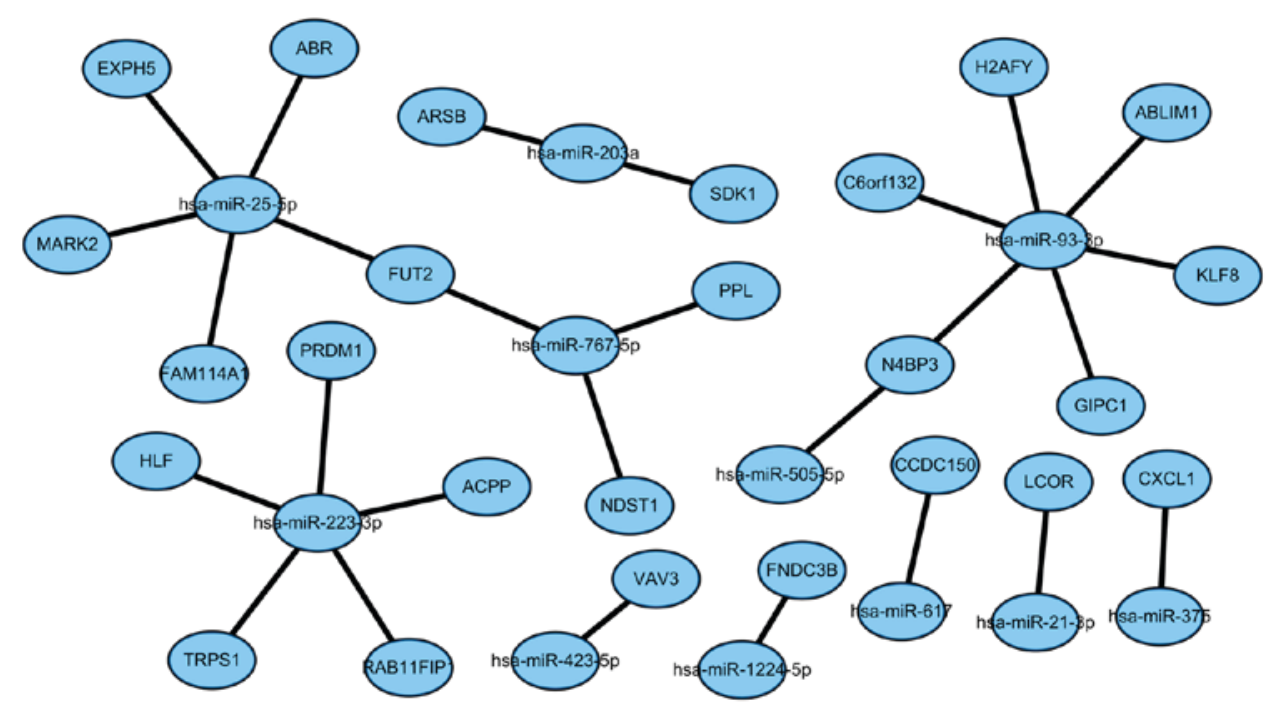

Figure 7. Final target genes of the differentially expressed microRNAs.
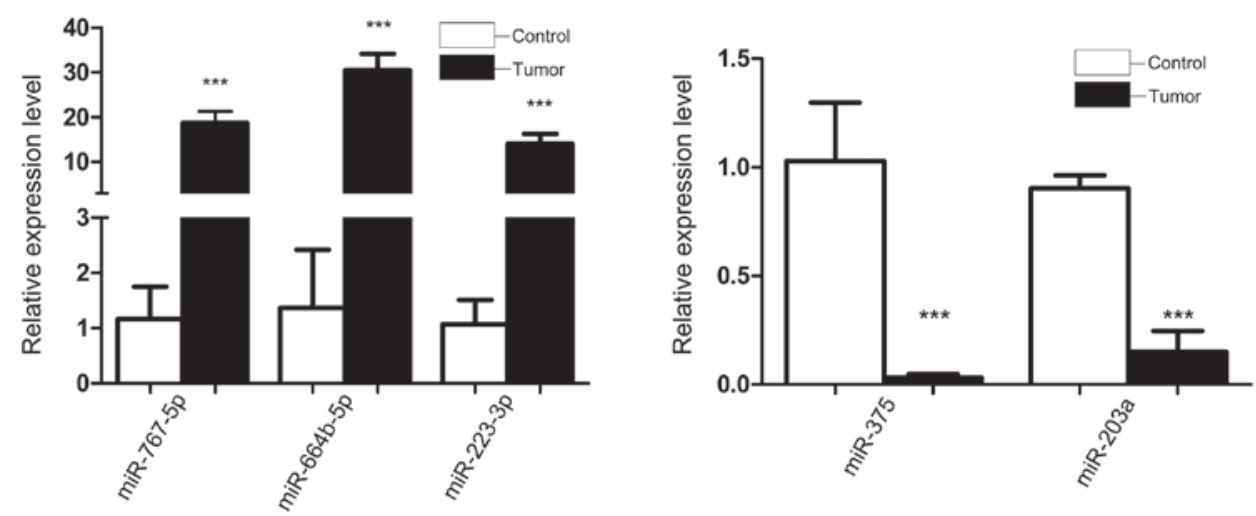

Figure 8. Differential expression of microRNAs between Kazakh esophagus squamous cell carcinoma and corresponding normal tissues was validated by reverse transcription-quantitative polymerase chain reaction. Data are presented as the mean \pm standard deviation $(\mathrm{n}=10)$. ${ }^{* * *} \mathrm{P}<0.001 \mathrm{vs}$. control.

The occurrence and development of esophageal cancer is associated with a number of factors. The most common risk factors of esophageal cancer include smoking, drinking and human papillomavirus infection. miRNAs are also widely involved in the progression of tumor formation (19). The effect of different risk factors and clinicopathological features on the expression of miRNA in esophageal cancer can be analyzed by gene chip technology, a method used to understand molecular mechanisms and provide diagnostic markers or new targets for therapy (44). Unfortunately, due to the limitation of the sample size in the current study, the correlations were not explored between the differential expression of miRNAs, clinicopathological characteristics and risk factors. Future studies should investigate these correlations by expanding the sample size.

The current study predicted the target genes of the differentially expressed miRNAs and then used bioinformatics to analyze the functions of these genes. The differentially expressed miRNAs may serve important roles by regulating these functions. Although the mechanisms of how the differentially expressed miRNAs contribute to the development of Kazakh ESCC are unknown, the findings of the current study provides the prerequisite for a future study to identify useful markers for diagnosing and treating Kazakh ESCC. Subsequent experiments are required to further clarify the roles and mechanisms of the differentially expressed miRNAs and their target genes in ESCC both in vivo and in vitro.

In conclusion, the current study performed a preliminary analysis of the biological effects of differentially expressed miRNAs in ESCC in Kazakh patients, which may be associated with the occurrence of the disease. Several potential target genes of these miRNAs were also identified. Studying these target genes can increase our understanding of the underlying mechanisms, which in turn can assist the discovery of improved uses of these differential miRNAs, possibly as markers of clinical diagnosis or therapeutic targets. The current findings can apply not only to all patients with ESCC, but also Kazakh patients with unique clinicopathological features. Further studies are needed to verify the role of these differentially expressed miRNAs in Kazakh ESCC.

\section{Acknowledgements}

Not applicable. 


\section{Funding}

The current study was supported by the National Natural Science Foundation of China (grant no. 81260362).

\section{Availability of data and materials}

All data generated or analyzed during this study are included in this published article.

\section{Authors' contributions}

YZ and WC conceived and designed the experiments. GS made substantial contributions to tissue sample collection. LC and GS performed the experiments. LC, GS, CF and GL analyzed the data. LC, CF and GL drafted and revised the manuscript. LC prepared the figures and tables. All authors read and approved the final manuscript.

\section{Ethics approval and consent to participate}

Ethics approval was obtained for the present study from the Ethics Committee of the First Affiliated Hospital of Medical College of Shihezi University and written informed consent was obtained from all patients.

\section{Patient consent for publication}

Not applicable.

\section{Competing interests}

The authors declare that they have no competing interests.

\section{References}

1. Li C, Xia G, Jianqing Z, Mei Y, Ge B and Li Z: Serum differential protein identification of Xinjiang Kazakh esophageal cancer patients based on the two-dimensional liquid-phase chromatography and LTQ MS. Mol Biol Rep 41: 2893-2905, 2014.

2. Torre LA, Bray F, Siegel RL, Ferlay J, Lortet-Tieulent J and Jemal A: Global cancer statistics, 2012. CA Cancer J Clin 65: 87-108, 2015.

3. Wang J, Jia Y, Wang N, Zhang X, Tan B, Zhang G and Cheng Y: The clinical significance of tumor-infiltrating neutrophils and neutrophil-to-CD8+ lymphocyte ratio in patients with resectable esophageal squamous cell carcinoma. J Transl Med 12: 7, 2014.

4. Zeng H, Zheng R, Guo Y, Zhang S, Zou X, Wang N, Zhang L, Tang J, Chen J, Wei K, et al: Cancer survival in China, 2003-2005: A population-based study. Int J Cancer 136: 1921-1930, 2015.

5. Zhang Y: Distribution of Esophageal Cancer in Xinjiang. J Xinjiang Med Univ 11: 139-145, 1988. (In Chinese)

6. Wang XM, Jie ES and Yan-Qing MA: Case-control study on risk factors of esophageal cancer in Xinjiang Kazakh nationality. Chinese J Public Health 23: 737-738, 2007. (In Chinese)

7. Kozomara A and Griffithsjones S: miRBase: Annotating high confidence microRNAs using deep sequencing data. Nucleic Acids Res 42: D68-D73, 2014.

8. Baek D, Villén J, Shin C, Camargo FD, Gygi SP and Bartel DP: The impact of microRNAs on protein output. Nature 455: 64-71, 2008.

9. Harada K, Baba Y, Ishimoto T, Shigaki H, Kosumi K, Yoshida N, Watanabe $\mathrm{M}$ and Baba $\mathrm{H}$ : The role of microRNA in esophagea squamous cell carcinoma. J Gastroenterol 51: 520-530, 2016.

10. Laganà $\mathrm{A}$, Russo $\mathrm{F}$, Sismeiro $\mathrm{C}$, Giugno R, Pulvirenti $\mathrm{A}$ and Ferro A: Variability in the incidence of miRNAs and genes in fragile sites and the role of repeats and $\mathrm{CpG}$ islands in the distribution of genetic material. PLoS One 5: e11166, 2010.
11. Friedman JM and Jones PA: MicroRNAs: Critical mediators of differentiation, development and disease. Swiss Med Wkly 139: 466-472, 2009.

12. Gao W, Shen H, Liu L, Xu J, Xu J and Shu Y: MiR-21 overexpression in human primary squamous cell lung carcinoma is associated with poor patient prognosis. J Cancer Res Clin Oncol 137: 557-566, 2011.

13. Hu JM, Chang AM, Chen YZ, Yuan XL and Li F: Regulatory Role of miR-203 in occurrence and progression of kazakh esophageal squamous cell carcinoma. Sci Rep 6: 23780, 2016.

14. Song X, You W, Zhu J, Cui X, Hu J, Chen Y, Liu W, Wang L, Li S, Wei Y, et al: A genetic variant in miRNA-219-1 is associated with risk of esophageal squamous cell carcinoma in chinese kazakhs. Dis Markers 2015: 541531, 2015.

15. Yamasaki M, Miyata H, Miyazaki Y, Takahashi T, Kurokawa Y, Nakajima K, Takiguchi S, Mori M and Doki Y: Evaluation of the nodal status in the 7th edition of the UICC-TNM classification for esophageal squamous cell carcinoma: Proposed modifications for improved survival stratification: Impact of lymph node metastases on overall survival after esophagectomy. Ann Surg Oncol 21: 2850-2856, 2014.

16. Zhao T and Liu H: 'Sparse Additive Machine', International Conference on Artificial Intelligence and Statistics, 2012.

17. Shannon P, Markiel A, Ozier O, Baliga NS, Wang JT, Ramage D, Amin N, Schwikowski B and Ideker T: Cytoscape: A software environment for integrated models of biomolecular interaction networks. Genome Res 13: 2498-2504, 2003.

18. Livak KJ and Schmittgen TD: Analysis of relative gene expression data using real-time quantitative PCR and the 2(-Delta Delta C(T)) method. Methods 25: 402-408, 2001.

19. Domper Arnal MJ, Ferrandez Arenas A and Lanas Arbeloa A: Esophageal cancer: Risk factors, screening and endoscopic treatment in Western and Eastern countries. World J Gastroenterol 21: 7933-7943, 2015

20. Guo Y, Chen Z, Zhang L, Zhou F, Shi S, Feng X, Li B, Meng X, Ma X, Luo M, et al: Distinctive microRNA profiles relating to patient survival in esophageal squamous cell carcinoma. Cancer Res 68: 26-33, 2008.

21. Lu J, Getz G, Miska EA, Alvarez-Saavedra E, Lamb J, Peck D, Sweet-Cordero A, Ebert BL, Mak RH, Ferrando AA, et al: MicroRNA expression profiles classify human cancers. Nature 435: 834-838, 2005.

22. Yang L, Ji Y, Chen L, Li M, Wu F, Hu J, Jiang J, Cui X, Chen Y, Pang L, et al: Genetic variability in LMP2 and LMP7 is associated with the risk of esophageal squamous cell carcinoma in the Kazakh population but is not associated with HPV infection. PLoS One 12: e0186319, 2017.

23. Zhou YX, Zhou KM, Liu Q, Wang H, Wang W, Shi Y and Ma YQ: The effect of Glut1 and c-myc on prognosis in esophageal squamous cell carcinoma of Kazakh and Han patients. Future Oncol 14: 1801-1815, 2018.

24. Li J, Li X, Li Y, Yang H, Wang L, Qin Y, Liu H, Fu L and Guan XY: Cell-specific detection of mir-375 downregulation for predicting the prognosis of esophageal squamous cell carcinoma by miRNA in situ hybridization. PLoS One 8: e53582, 2013.

25. Huang G: Erratum: Differential expression of miR-21 and miR-75 in esophageal carcinoma patients and its clinical implication. Am J Transl Res 9: 1961, 2017.

26. Wang C, Guan S, Chen X, Liu B, Liu F, Han L, Un Nesa E, Song Q, Bao C, Wang X and Cheng Y: Clinical potential of miR-3651 as a novel prognostic biomarker for esophageal squamous cell cancer. Biochem Biophys Res Commun 465: 30-34, 2015.

27. Tian L, Li M, Ge J, Guo Y, Sun Y, Liu M and Xiao H: MiR-203 is downregulated in laryngeal squamous cell carcinoma and can suppress proliferation and induce apoptosis of tumours. Tumour Biol 35: 5953-5963, 2014.

28. Liu Y, Ren F, Rong M, Luo Y, Dang Y and Chen G: Association between underexpression of microrna-203 and clinicopathological significance in hepatocellular carcinoma tissues. Cancer Cell Int 15: 62, 2015.

29. Mao L, Zhang Y, Mo W, Yu Y and Lu H: BANF1 is downregulated by IRF1-regulated microRNA-203 in cervical cancer. PLoS One 10: e0117035, 2015.

30. Wang N, Liang H, Zhou Y, Wang C, Zhang S, Pan Y, Wang Y, Yan X, Zhang J, Zhang CY, et al: miR-203 suppresses the proliferation and migration and promotes the apoptosis of lung cancer cells by targeting SRC. PLoS One 9: e105570, 2014.

31. Li Y, Tan W, Neo TW, Aung MO, Wasser S, Lim SG and Tan TM: Role of the miR-106b-25microRNA cluster in hepatocellular carcinoma. Cancer Sci 100: 1234-1242, 2009. 
32. Yang M, Liu R, Sheng J, Liao J, Wang Y, Pan E, Guo W, Pu Y and Yin L: Differential expression profiles of microRNAs as potential biomarkers for the early diagnosis of esophageal squamous cell carcinoma. Oncol Rep 29: 169-176, 2013.

33. Liu T, Liu Q, Zheng S, Gao X, Lu M, Yang C, Dai F, Sheyhidin I and Lu X: MicroRNA-21 promotes cell growth and migration by targeting programmed cell death 4 gene in Kazakh's esophageal squamous cell carcinoma. Dis Markers 2014: 232837, 2014

34. Savita U and Karunagaran D: MicroRNA-106b-25 cluster targets $\beta$-TRCP 2 , increases the expression of Snail and enhances cell migration and invasion in H1299 (non small cell lung cancer) cells. Biochem Biophys Res Commun 434: 841-847, 2013

35. Li BS, Zuo QF, Zhao YL, Xiao B, Zhuang Y, Mao XH, Wu C, Yang SM, Zeng H, Zou QM and Guo G: MicroRNA-25 promotes gastric cancer migration, invasion and proliferation by directly targeting transducer of ERBB2, 1 and correlates with poor survival. Oncogene 34: 2556-2565, 2015

36. Zhao Z, Liu J, Wang C, Wang Y, Jiang Y and Guo M: MicroRNA-25 regulates small cell lung cancer cell development and cell cycle through cyclin E2. Int J Clin Exp Pathol 7: 7726-7234, 2014

37. Qu J, Li M, Zhong W and Hu C: Prognostic role of microRNA-25 in cancers: Evidence from a meta-analysis. Int J Clin Exp Med 8: 12921-12927, 2015.

38. Xu X, Chen Z, Zhao X, Wang J, Ding D, Wang Z, Tan F, Tan X, Zhou F, Sun J, et al: MicroRNA-25 promotes cell migration and invasion in esophageal squamous cell carcinoma. Biochem Biophys Res Commun 421: 640-645, 2012.

39. Qian J, Li R, Wang YY, Shi Y, Luan WK, Tao T, Zhang JX, Xu YC and You YP: MiR-1224-5p acts as a tumor suppressor by targeting CREB1 in malignant gliomas. Mol Cell Biochem 403: 33-41, 2015.

40. Huang H, Jiang Y, Wang Y, Chen T, Yang L, He H, Lin Z, Liu T, Yang T, Kamp DW, Wu B and Liu G: miR-5100 promotes tumor growth in lung cancer by targeting Rab6. Cancer Lett 362: 15-24, 2015.

41. Matamala N, Vargas MT, Gonzalez-Campora R, Miñambres R, Arias JI, Menéndez P, Andrés-León E, Gómez-López G, Yanowsky K, Calvete-Candenas J, et al: Tumor MicroRNA expression profiling identifies circulating MicroRNAs for early breast cancer detection. Clin Chem 61: 1098-1106, 2015.

42. Song W, Tang L, Xu Y, Xu J, Zhang W, Xie H, Wang S and Guan X: Corrigendum: PARP inhibitor increases chemosensitivity by upregulating miR-664b-5p in BRCA1-mutated triple-negative breast cancer. Sci Rep 7: 44346, 2017.

43. Zha R, Guo W, Zhang Z, Qiu Z, Wang Q, Ding J, Huang S, Chen T, $\mathrm{Gu}$ J, Yao $\mathrm{M}$ and $\mathrm{He} \mathrm{X}$ : Genome-wide screening identified that miR-134 acts as a metastasis suppressor by targeting integrin $\beta 1$ in hepatocellular carcinoma. PLoS One 9: e87665, 2014.
44. Zang W, Wang Y, Du Y, Xuan X, Wang T, Li M, Ma Y, Li P, Chen $X$, Dong $Z$ and Zhao G: Differential expression profiling of microRNAs and their potential involvement in esophageal squamous cell carcinoma. Tumour Biol 35: 3295-3304, 2014.

45. Hakomori S: Aberrant glycosylation in cancer cell membranes as focused on glycolipids: Overview and perspectives. Cancer Res 45: 2405-2414, 1985.

46. Alikhani N, Ferguson RD, Novosyadlyy R, Gallagher EJ, Scheinman EJ, Yakar S and LeRoith D: Mammary tumor growth and pulmonary metastasis are enhanced in a hyperlipidemic mouse model. Oncogene 32: 961-967, 2013.

47. Berridge MJ, Lipp P and Bootman MD: The versatility and universality of calcium signalling. Nat Rev Mol Cell Biol 1: 11-21, 2000.

48. Zhao P, Xiao L, Peng J, Qian YQ, and Huang CC: Exosomes derived from bone marrow mesenchymal stem cells improve osteoporosis through promoting osteoblast proliferation via MAPK pathway. Eur Rev Med Pharmacol Sci 22: 3962-3970, 2018.

49. Weyant MJ, Carothers AM, Bertagnolli ME and Bertagnolli MM: Colon cancer chemopreventive drugs modulate integrin-mediated signaling pathways. Clin Cancer Res 6: 949-956, 2000.

50. Choi S and Anderson RA: IQGAP1 is a phosphoinositide effector and kinase scaffold. Adv Biol Regul 60: 29-35, 2016.

51. Wang XX, Wang K, Li XZ, Zhai LQ, Qu CX, Zhao Y, Liu ZR, Wang HZ, An QJ, Jing LW and Wang XH: Targeted knockdown of IQGAP1 inhibits the progression of esophageal squamous cell carcinoma in vitro and in vivo. PLoS One 9: e96501, 2014.

52. Xu CQ, Zhu ST, Wang M, Guo SL, Sun XJ, Cheng R, Xing J, Wang WH, Shao LL and Zhang ST: Pathway analysis of differentially expressed genes in human esophageal squamous cell carcinoma. Eur Rev Med Pharmacol Sci 19: 1652-1661, 2015.

53. Vij M, Shanmugam NP, Reddy MS, Sankaranarayanan S and Rela M: Paediatric hepatocellular carcinoma in tight junction protein 2 (TJP2) deficiency. Virchows Arch 471: 679-683, 2017.

54. Ganguly P and Ganguly N: Transcriptomic analyses of genes differentially expressed by high-risk and low-risk human papilloma virus E6 oncoproteins. Virusdisease 26: 105-116, 2015.

55. Zhang G, Zhang W, Li B, Stringer-Reasor E, Chu C, Sun L, Bae S, Chen D, Wei S, Jiao K, et al: MicroRNA-200c and microRNA141 are regulated by a FOXP3-KAT2B axis and associated with tumor metastasis in breast cancer. Breast Cancer Res 19: 73, 2017.

This work is licensed under a Creative Commons Attribution-NonCommercial-NoDerivatives 4.0 International (CC BY-NC-ND 4.0) License. 